\title{
La orientación sexual en personas mayores: Categorías, deseos y realidades
}

\section{Sexual orientation in older people: Categories, desires and realities}

Fecha de recepción: 27-07-2020

Fecha de aceptación: 16-11-2020

\author{
María Dolores Gil-Llario \\ Catedrática de Universidad. \\ Departamento de Psicología Evolutiva y de la Educación. \\ Facultad de Psicología. Universitat de València. España \\ Rafael Ballester-Arnal \\ Catedrático de Universidad. \\ Área de Personalidad, Evaluación y Tratamientos Psicológicos. \\ Facultad de Ciencias de la Salud. Universitat Jaume I de Castelló. España \\ Raquel Flores-Buils \\ Profesora Contratada Doctora. \\ Área de Psicología Evolutiva y de la Educación. \\ Facultad de Ciencias de la Salud. Universitat Jaume I de Castelló. España
}

\section{resumen/abstract:}

La diversidad sexual tiende a considerarse una cuestión que afecta principalmente a personas jóvenes por lo que los estudios centrados en la vivencia de una orientación sexual minoritaria en personas mayores son muy escasos. El objetivo de este estudio ha sido analizar el grado de coincidencia entre la orientación sexual autodefinida, el objeto de deseo y las personas con las que se han tenido relaciones sexuales. En este estudio han participado 244 personas (47,5\% hombres y 52,5\% mujeres) de entre 55 y 80 años, pertenecientes a distintos grupos de nivel socioeconómico, grado académico y situación laboral. Todas ellas han cumplimentado un cuestionario sobre orientación sexual y aspectos como religión o actividad sexual. Los resultados muestran que el 13\% de los mayores (8,6\% hombres, 16,4\% mujeres) no se sienten atraídos exclusivamente por el sexo opuesto, y el $6 \%$ reconoce haber mantenido prácticas homoeróticas (3,5\% hombres y 7,9\% mujeres). En los hombres la coincidencia entre autodefinición y objeto de deseo es mayor que en las mujeres. Se discute la necesidad de realizar futuros estudios cualitativos que posibiliten conocer qué variables, relacionadas con la historia sexual y la trayectoria vital de las personas, influyen en la exploración y/o aceptación de opciones sexuales no mayoritarias.

Sexual diversity tends to be considered as an issue that mainly affects young people, so studies focusing on the experience of a minority sexual orientation in older people are very scarce. The aim of this study was to analyse the degree of coincidence between the self-defined sexual orientation, the object of desire and the people with whom one has had sexual relations. A total of 244 people (47.5\% men and $52.5 \%$ women) between the ages of 55 and 80 participated in this study, belonging to different groups of socioeconomic level, academic degree and employment situation. All of them completed a questionnaire on sexual orientation and aspects such as religion or sexual activity. The results show that 13 per cent of older people (8.6 per cent men, 16.4 per cent women) are not attracted exclusively to the opposite sex, and 6 per cent acknowledge having maintained homoerotic practices $(3.5$ per cent men, 7.9 per cent women). In men the coincidence between self-definition and object of desire is greater than in women. It discusses the need for future qualitative studies that make it possible to know which variables, related to the sexual history and life trajectory of individuals, influence the exploration and/or acceptance of non-majority sexual options.

\section{palahras clave/keywords:}

orientación sexual, personas mayores, género, atracción sexual, relaciones sexuales. sexual orientation, older people, gender, sexual attraction, sexual intercourse. 


\section{Introducción}

La orientación sexual tradicionalmente se ha definido como el deseo o atracción sexual que se tiene hacia el mismo sexo o hacia el otro sexo. En este sentido se han propuesto tres categorías para etiquetar las diferentes orientaciones del deseo sexual, a saber, la heterosexualidad, la bisexualidad y la homosexualidad. Kinsey fue uno de los primeros en destacar que la orientación sexual no podía ser considerada categorialmente, sino como un continuo que abarcaría desde sentirse atraído exclusivamente por el otro sexo hasta sentirse atraído exclusivamente por el mismo sexo (Kinsey, Pomeroy y Martin, 1948). Más tarde Klein (1978) aumentó la complejidad al indicar que el continuo propuesto por Kinsey, debía atenderse no solo en lo referido a la atracción sexual, sino también a si realmente se han llevado a cabo comportamientos en esa línea, o si se han deseado o se ha fantaseado con ello, independientemente de si se han realizado o no. Además, enfatiza que esos continuos son dinámicos y cambiantes en el tiempo. Gracias en gran parte a estos autores, hoy consideramos que la orientación sexual es un continuo dimensional en el que se sitúan todos los individuos de manera dinámica, alejado de las categorías excluyentes y absolutas. Sin embargo, el planteamiento categorial de la orientación del deseo sexual, alejado de la realidad y del rigor científico, sigue imperando en nuestras mentes, y tal y como indican diversos trabajos (Ballester-Arnal y Gil-Llario, 2016; Ballester-Arnal y Gil-Llario, 2019), gran parte de los estudios se refieren a estas categorías.

En el abordaje del estudio de la orientación sexual en las personas mayores debemos tener en cuenta esta dimensionalidad, pero sobre todo, debemos entender que la complejidad de la construcción de la identidad en su caso es mucho mayor por las dificultades derivadas de los factores sociopolíticos que marcaron su desarrollo en las etapas más tempranas. El estigma estructural con el que se vieron obligados a crecer las personas con una orientación alejada de la aceptada, desgraciadamente fue una característica común en muchos países, si bien en unos más que en otros. En un estudio reciente, Pachankis y Bränström (2018) examinaron la asociación entre el estigma estructural, la ocultación de la identidad y la satisfacción vital de las minorías sexuales en 85.582 adultos de minorías sexuales procedentes de 28 países europeos. El estigma estructural fue evaluado mediante la existencia de leyes, políticas y actitudes nacionales que afectan a las minorías sexuales en cada país. Los resultados obtenidos fueron que, a nivel de país, el estigma estructural explicó el 60\% de la varianza en satisfacción de vida y más del 70\% de la varianza en el ocultamiento de la orientación sexual, siendo esta variable la mediadora entre el estigma estructural y la satisfacción de la vida. En los países con un elevado estigma, la ocultación protegía contra una satisfacción vital aún menor en tanto que también protegía parcialmente contra la discriminación y victimización (Pachankis y Bränström, 2018).

Nuestro país no ha sido una excepción. La heteronormatividad, la norma imperante en buena parte de nuestra historia reciente, enseña que la única forma normal de entablar relaciones sexuales y románticas es ser heterosexual, a menudo borrando otras posibilidades. La heteronormatividad prescrita externamente, que señala la idea de que uno debe ser heterosexual, heterorromántico y monógamo, puede contribuir a la angustia que experimentan los 
individuos no heterosexuales cuando se cuestionan su orientación sexual, cosa que probablemente, ocurrió con cierta frecuencia entre las personas que construyeron su identidad sexual hace 4 o 5 décadas (Alonso-Sánchez y Muyor-Rodríguez, 2020). La teoría de la incertidumbre-identidad (Hogg, 2007, 2012) afirma que el cuestionamiento de las identidades previas causa angustia; para reducir esta angustia, los individuos que se cuestionan, gravitan hacia nuevas identidades sociales con una gran capacidad de atracción, es decir, grupos bien definidos y homogéneos. Boyer y Lorenz (2020) intentaron comprobar si dicha teoría era aplicable en el contexto del desarrollo de la orientación sexual. Para ello aplicaron una encuesta online a 514 individuos no heterosexuales en la que evaluaban cómo los ideales heteronormativos, la identidad y la autenticidad de la etiqueta se relacionaban con la angustia cuando uno se cuestiona la orientación sexual. Boyer y Lorenz (2020) hallaron que los ideales heteronormativos fuertemente prescritos constituyen un predictor significativo de la angustia que se siente al cuestionarse la orientación sexual. La existencia de ideales heteronormativos puede contribuir a un cuestionamiento más angustioso de la orientación sexual y puede impedir que la información sobre identidades alternativas no heterosexuales sea escuchada. En el estudio de Boyer y Lorenz (2020) la atracción por el grupo de orientación sexual no fue un predictor significativo de la incertidumbre relacionada con la angustia, lo que sugiere que la teoría de la incertidumbre-identidad puede no aplicarse totalmente a la crisis de identidad causada por la orientación sexual. Las personas que se han cuestionado su orientación en edades avanzadas no tienden a recurrir a esos grupos claramente definidos, los cuales pueden incomodarles.

Las personas mayores vivieron unas circunstancias socio-políticas que determinaron el clima familiar en el que crecieron, el cual pudo dificultar en gran medida la construcción de la identidad en los casos en que la orientación sexual no era la heteronormativa. Por este motivo, se ha hipotetizado la existencia de lazos intergeneracionales más débiles entre los padres y sus hijas e hijos adultos en relaciones del mismo sexo. En un reciente estudio llevado a cabo en los Países Bajos, Fischer y Kalmijn (2020) analizaron hasta qué punto los lazos intergeneracionales eran más débiles entre los padres y sus hijas e hijos adultos en relaciones del mismo sexo, y si la fuerza de estos lazos podía deberse a las diferencias de género y a las opiniones tradicionales de los padres cuando el/la niño/a estaba creciendo, informadas retrospectivamente. Encontraron que las personas que están en relaciones del mismo sexo en la edad adulta se habían ido de casa antes y se habían alejado más de sus padres que los que tenían relaciones con personas de sexo diferente. Se habían ido generalmente debido a conflictos en casa, y con menos frecuencia para mudarse directamente con un compañero. Ya en la edad adulta, los hombres que tienen relaciones con hombres tienen vínculos más ambivalentes con sus madres y más frágiles con sus padres que los hombres que tienen relaciones con mujeres. En el caso de las mujeres que tienen relaciones con mujeres el vínculo con su padre es, en promedio, más débil sobre todo cuando el hogar paterno había sido más tradicional en términos de división de roles de género en la crianza de los hijos. Las mujeres que tienen relaciones con mujeres también presentan un contacto menos frecuente con ambos padres en comparación con las mujeres que tienen relaciones con hombres (Fischer y Kalmijn, 2020). Observando estos datos obtenidos en 2020 y en los Países Bajos pode- 
mos imaginar lo que pudo suponer hace unas décadas tener una relación con personas del mismo sexo para los hogares españoles con los valores sociales y religiosos que en aquellos momentos imperaban. Asumir una orientación no normativa podía conllevar, con una elevada probabilidad, una ruptura dolorosa con la familia nuclear, por lo que cabe esperar que muchas personas abortaran, o al menos mantuvieran bajo control y en secreto, sus deseos hasta edades bien avanzadas viéndose privados de la libertad para experimentar e incluso para considerar una opción poco aceptada socialmente (Ceballos-Fernández, 2014). Con el tiempo y la flexibilización de las normas, sobre todo para los más jóvenes, ha ido siendo progresivamente menos complicado, si bien todavía dista mucho de ser sencillo, cuestionar la opción imperante, pero para las personas mayores en muchos casos esta apertura llegó tarde. Es por esto que solo las personas con determinada historia y características son capaces de identificarse públicamente como personas homosexuales o bisexuales, siendo mucho más frecuente que, aun admitiendo que tienen o han tenido deseo por mantener relaciones sexuales con personas de su mismo sexo, no por ello se etiquetan a sí mismos como "homosexuales" o como "bisexuales".

Esta conflictividad interior de la que antes hablábamos que caracteriza a quienes no son heterosexuales es, si cabe, mayor entre las personas bisexuales ya que éstas pueden experimentar una binegatividad generalizada proveniente tanto de quienes se definen como heterosexuales como de las personas homosexuales como resultado de diversos factores psicosociales y relacionales. Arriaga y Parent (2019) evaluaron los niveles de binegatividad experimentada e internalizada en 350 hombres y mujeres estadounidenses autoidentificados como bisexuales. Los resultados del análisis de regresión indicaron que la binegatividad de las personas homosexuales, pero no la de las heterosexuales, se asociaba significativa y positivamente con la binegatividad internalizada. Una interacción significativa entre la binegatividad de personas homosexuales y el género de la pareja reveló una asociación más fuerte entre aquellos en relaciones del mismo género, de tal manera que quienes tenían parejas del mismo género e indicaban mayor binegatividad proveniente de personas homosexuales experimentaron una mayor binegatividad internalizada que los que tenían parejas de otro género. Es decir, las personas bisexuales son mal vistas tanto por quienes solo tienen sexo con personas de su mismo sexo como por los que tienen con personas del otro sexo, y si cabe todavía peor por los autoidentificados como homosexuales llegando a internalizar dicha binegatividad (Arriaga y Parent, 2019). Esto es especialmente problemático en el caso de las personas mayores que, habiendo visto tan cercenadas sus posibilidades de elección han desarrollado sus vidas con parejas del otro sexo quienes pueden atraerles tanto como parejas del mismo sexo.

Otra variable fundamental en este contexto es la religiosidad. En un estudio llevado a cabo por Moscardini, Douglass, Conlin, y Duffy (2018) con 365 personas bisexuales encontraron que las expectativas de rechazo y la bifobia internalizada, y no la discriminación, tenían importantes efectos negativos directos sobre el significado de la vida. Sin embargo, la discriminación tuvo efectos indirectos negativos. La religiosidad moderó el vínculo discriminación-significado de la vida de tal manera que las personas con mayor religiosidad experimentaron reducciones en el significado de la vida debido a una mayor discriminación (Moscardini et al., 2018). 
Pese a todos estos estudios realizados con población adulta general, la diversidad sexual ha sido uno de los aspectos más invisibilizados, como consecuencia de la heteronormatividad imperante (Herz \& Johansson, 2015). El hecho de que la orientación sexual de los adultos mayores sea un tema escasamente estudiado (Fredtiksen-Goldsen \& Kim, 2015), subraya la necesidad de analizar en profundidad el fenómeno de las orientaciones sexuales no heterosexuales en hombres y mujeres mayores, comparando su autoadscripción con sus deseos y comportamientos reales a la luz de algunas variables que la literatura ha mostrado relevantes como la religión.

\section{Método}

\section{Participantes}

En este estudio han participado 244 adultos (47,5\% hombres y 52,5\% mujeres) de edades comprendidas entre los 55 y 80 años de edad. Por lo que se refiere a la relación de pareja, el $72,9 \%$ están casados o tienen pareja. El 27,1\% restante no viven en el momento de la evaluación en pareja, ya sea por estar separados o divorciados $(10,7 \%)$, ser viudos/as $(13,5 \%)$ o solteros/as $(2,9 \%)$. El nivel de estudios se reparte de forma bastante equitativa entre los tres niveles educativos, ya que el 20,1\% tienen estudios primarios, el 38,9\% estudios secundarios y el 39,4\% estudios superiores, existiendo solo un 1,6\% sin estudios. En cuanto a la situación laboral, el 16,3\% son trabajadores en activo, el 71,2\% son jubilados/as, el 7,1\% se dedica a las tareas del hogar y solo el 5,4\% están en situación de desempleo. La orientación política del grupo de participantes también se reparte de forma bastante homogénea a lo largo del abanico de opciones ideológicas que van desde la izquierda hasta la derecha en 5 categorías. El 19,9\% se define como de izquierdas, el 29\% de centro-izquierda, el 33,7\% de centro, el $13,7 \%$ de centro-derecha y el 3,7\% de derechas. Por lo que respecta a la religión, el $50,4 \%$ se define como creyente no practicante, el $18 \%$ como creyentes practicantes y el $31,6 \%$ como ateos o agnósticos. A excepción de dos personas que dicen ser Testigo de Jehová y universalista respectivamente, todos los demás afirman ser católicos.

\section{Procedimiento}

Para la realización del estudio se ha reclutado la muestra entre los estudiantes de la Universitat per a Majors de la Universitat Jaume I de Castelló. La composición de la Universitat per a Majors garantiza la heterogeneidad de la muestra en cuanto a nivel socioeconómico, grado académico y situación laboral, como hemos podido comprobar. Para la participación en el estudio se informó de la naturaleza del proyecto especificando sus objetivos y la finalidad con la que iban a ser tratados los datos solicitando la colaboración voluntaria, anónima y confidencial de todas las personas. Tras la obtención del consentimiento informado, las personas interesadas en participar cumplimentaron una batería de instrumentos que fue administrada tanto en formato online como presencial. El tiempo de administración fue entre 10 y 15 minutos.

Para la opción online, se habilitó una plataforma virtual a través de la cual las personas participantes pudieron acceder a los instrumentos, así como a un documento informativo previo y al formulario del consentimiento informado. En la opción presencial, las personas participantes cumplimentaron los instrumentos en la sala de usos múltiples de los centros 
participantes, así como en el laboratorio que el equipo SALUSEX tiene en la Universitat Jaume I de Castellón. Los instrumentos fueron adaptados debidamente para facilitar su acceso a las personas con problemas visuales. En este artículo se analiza la selección de ítems que hacen referencia a los aspectos sociodemográficos así como los relativos a su orientación sexual.

\section{Instrumento}

Para la realización de este estudio se ha utilizado una selección de ítems del Cuestionario de sexualidad para personas mayores (CSPM). Este instrumento, desarrollado ad hoc, reúne 4 apartados: sociodemográfica, historia médica, prácticas sexuales y estrategias de adaptación sexual. Para este trabajo se han utilizado dos de las áreas exploradas en este cuestionario, la Información sociodemográfica: compuesta de 6 ítems (edad, estado civil, nivel de estudios, situación laboral, creencias religiosas e ideología política), y las Prácticas sexuales actuales: que incluye, a través de 10 ítems, cuestiones relativas a la orientación sexual, a las parejas sexuales y románticas que se han tenido a lo largo de la vida, el uso de material pornográfico, la contratación de servicios sexuales (trabajadores y trabajadoras sexuales) y las fantasías sexuales. Estos 16 ítems tienen diferentes formatos de respuesta dependiendo del contenido: escala con distintas frecuencias u opciones, y preguntas dicotómicas con respuesta sí/no.

Además, para conocer la diversidad afectivo-sexual de las personas mayores utilizamos la escala Kinsey de siete alternativas, que van desde "Me siento atraído/a solo por personas del sexo opuesto" a "Me siento atraído/a solo por personas del mismo sexo" pasando por 5 opciones intermedias. Se introdujo una opción que no estaba en la escala de Kinsey para recoger la asexualidad: "no me siento atraído por ningún sexo".

\section{Análisis de datos}

Se realizaron análisis descriptivos de las principales variables estudiadas. Asimismo, para analizar las diferencias entre hombres y mujeres, se realizaron pruebas de Chi cuadrado.

\section{Resultados}

\section{Diversidad afectivo-sexual}

Nuestros resultados muestran que un $87,3 \%$ de la muestra dijo sentirse sólo atraída por personas del sexo opuesto; un $8,6 \%$ principalmente por personas del sexo opuesto; un $0,6 \%$ un poco más por personas del sexo opuesto; nadie contestó que se sentía atraído indiferentemente por ambos sexos ni un poco más por personas del mismo sexo; un 0,8\% se sentía atraída principalmente por personas del mismo sexo; y un $0,4 \%$ únicamente por personas del mismo sexo. Finalmente, un $2 \%$ afirmó que no se sentía atraído/a por ningún sexo. Como se puede apreciar, al evaluar la orientación sexual de este modo se observa que un $12,7 \%$ de las personas mayores no se sienten atraídas exclusivamente por el sexo opuesto, si bien cuando se les pregunta por su adscripción el porcentaje de personas que se autodefinen como heterosexuales es mayor $(96,7 \%)$. 
Los resultados desagregados para hombres y mujeres se presentan en la Figura 1. Obsérvese que en esta muestra prácticamente todos los hombres se quedan representados en las dos primeras opciones de respuesta: atracción heterosexual exclusiva con el 91,4\% o principal con el 6,9\%, mientras que la orientación sexual de las mujeres se distribuye más a lo largo del continuo siendo sólo un $83,6 \%$ las que se consideran exclusivamente atraídas por el sexo opuesto. También es un porcentaje ligeramente superior el de mujeres que no se sienten atraídas por ningún sexo $(2,3 \%)$ que el de hombres $(1,7 \%)$. Con todo, las diferencias entre hombres y mujeres no resultan ser estadísticamente significativas $\left(\mathrm{Chi}^{2}=5,819, \mathrm{p} \leq 0,324\right)$.

Figura 1. Orientación de la atracción sexual de hombres y mujeres siguiendo la escala Kinsey (ampliada) (\%)

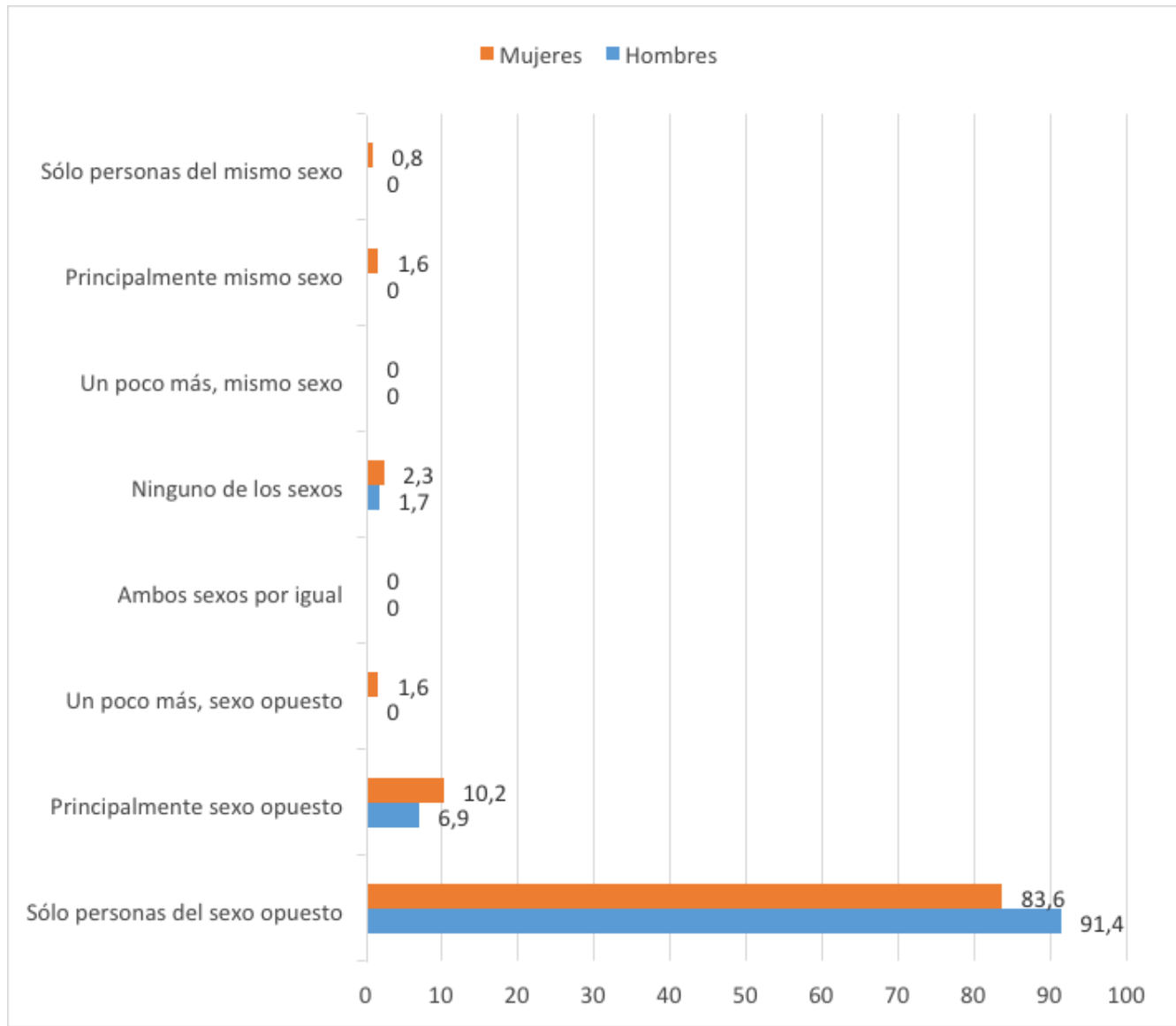




\section{Atracción sexual}

El segundo nivel de análisis consistió en ver si existen diferencias en cuanto a los porcentajes entre el haber sentido atracción y el haber llevado a la realidad dicha atracción teniendo relaciones sexuales con las personas que son el objeto de atracción. Si observamos el caso de los hombres (ver figura 2) vemos que, en el caso de la atracción exclusiva por el sexo opuesto, es mayor el porcentaje de hombres que han tenido relaciones sexuales con personas del sexo opuesto, que el de hombres que se sienten atraídos exclusivamente por el sexo opuesto $(96,5 \%$ frente a $91,4 \%)$. La segunda opción, que guarda una distancia notable con la primera en cuanto a porcentaje, es la que señalan quienes tienen la heterosexualidad como principal opción, pero no como exclusiva. El 6,9\% no desea a las personas del sexo opuesto de forma exclusiva, es decir, también muestra algún deseo no heterosexual pero solo el 2,6\% ha tenido alguna relación no heterosexual. La siguiente opción escogida, y en un porcentaje menor todavía $(1,7 \%)$ ya es la de la asexualidad que, como cabía esperar, no se ve acompañada por relaciones sexuales.

Figura 2. Orientación de la atracción sexual y de las relaciones sexuales de hombres siguiendo la escala Kinsey (ampliada) (\%)

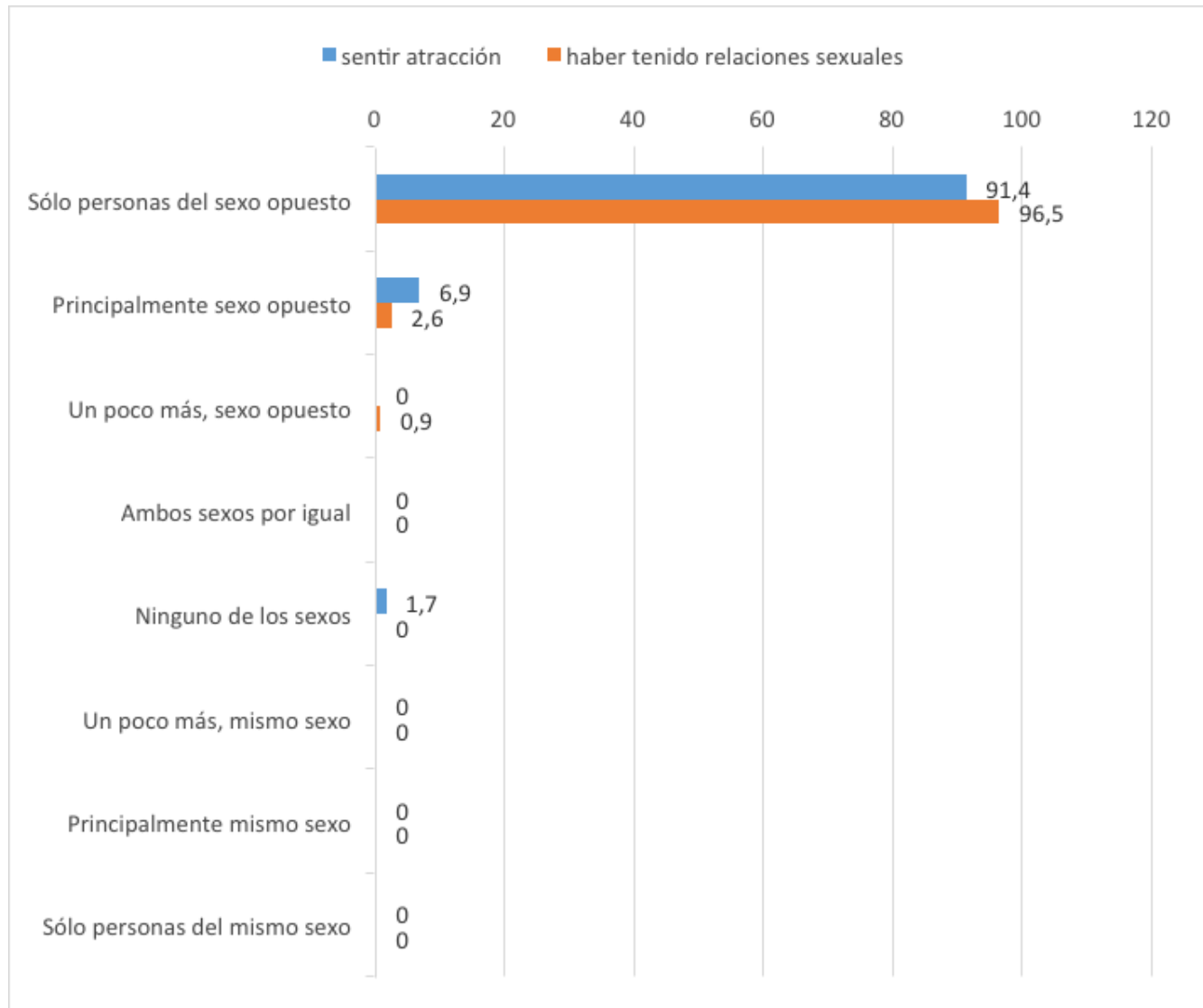


Analizando estos mismos aspectos, en el caso de las mujeres encontramos (ver figura 3) un patrón semejante en cuanto a que se han tenido más relaciones sexuales con personas exclusivamente del sexo opuesto de las que se han deseado $(92,1 \%$ frente a $83,6 \%)$. Pese a esto, encontramos diferencias en la proporción de personas exclusivamente heterosexuales $\mathrm{y}$, sobre todo, en el reparto a lo largo del continuo, siendo mucho mayor en su caso. De los 8 niveles, solo uno recoge porcentajes nulos. Un 11,8\% de las mujeres admiten sentirse atraídas también por personas del mismo sexo, y un $4,8 \%$ han llevado a cabo relaciones sexuales en este sentido. Yendo más allá, un 4,7\% se han sentido atraídas en mayor o menor medida por personas de su mismo sexo, teniendo relaciones un $2,4 \%$. Por último, un pequeño porcentaje $(2,3 \%)$ señala no sentir atracción por ninguno de los dos sexos y solo un $0,8 \%$ no ha tenido relaciones con ninguno de los dos sexos. Sin embargo, de nuevo, las diferencias entre hombres y mujeres no resultan estadísticamente significativas $\left(\mathrm{Chi}^{2}=5,620, \mathrm{p} \leq 0,467\right)$.

Figura 3. Orientación de la atracción sexual y de las relaciones sexuales de mujeres siguiendo la escala Kinsey (ampliada) (\%)

\begin{tabular}{|c|c|c|c|c|c|c|c|c|c|c|}
\hline & \multicolumn{2}{|c|}{ m sentir deseo } & \multicolumn{4}{|c|}{ haber tenido relaciones } & \multirow[b]{2}{*}{70} & \multirow[b]{2}{*}{80} & \multirow{2}{*}{\multicolumn{2}{|c|}{100}} \\
\hline & 0 & 20 & 30 & 40 & 50 & 60 & & & & \\
\hline Sólo personas del sexo opuesto & & & & & & & & & 83,6 & 92.1 \\
\hline Principalmente sexo opuesto & 4 & 0,2 & & & & & & & & \\
\hline Un poco más, sexo opuesto & $\int_{0}^{1,6}$ & & & & & & & & & \\
\hline Ambos sexos por igual & $\begin{array}{l}0 \\
0\end{array}$ & & & & & & & & & \\
\hline Ninguno de los sexos & $\left.\right|_{0,8} ^{2,3}$ & & & & & & & & & \\
\hline Un poco más, mismo sexo & $\begin{array}{l}0 \\
0,8\end{array}$ & & & & & & & & & \\
\hline Principalmente mismo sexo & $\begin{array}{l}1,6 \\
0,8\end{array}$ & & & & & & & & & \\
\hline Sólo personas del mismo sexo & $\begin{array}{l}0,8 \\
0,8\end{array}$ & & & & & & & & & \\
\hline
\end{tabular}




\section{Orientación sexual}

También se les preguntó cómo se categorizarían a sí mismos por lo que se refiere a su orientación sexual. En la tabla 1 se puede observar el grado de coincidencia entre cómo se perciben, qué les atrae y con qué tipo de personas han tenido relaciones. Como puede observarse por lo que se refiere a quienes se definen como heterosexuales, si bien no se trata de un grupo que de forma compacta solo se sienta atraído por personas del sexo opuesto, lo cierto es que nadie ha tenido relaciones con personas de su mismo sexo. Pero no ocurre lo mismo con quienes se identifican como homosexuales que se han sentido atraídos y han tenido relaciones con personas de su mismo sexo en la misma medida que con personas del sexo opuesto. Más sorprendentes son los resultados en relación con quienes se identifican como bisexuales: el $50 \%$ dice haberse sentido atraído únicamente por personas del sexo opuesto

Tabla 1. Porcentajes cruzados entre "sentir atracción hacia", "haber tenido relaciones con" y "autoidentificarse como"

\begin{tabular}{lccccc}
\hline & & Heterosexual & Homosexual & Bisexual & Asexual \\
\hline Ninguna & Atracción & 1,3 & 0 & 0 & 50 \\
persona & Relación & 0,4 & 0 & 0 & 0 \\
Solo sexo & Atracción & 89,2 & 50 & 50 & 50 \\
opuesto & Relación & 95,7 & 50 & 50 & 50 \\
Principalmente & Atracción & 8,6 & 0 & 0 & 0 \\
sexo opuesto & Relación & 3,5 & 0 & 0 & 50 \\
Un poco más & Atracción & 0,9 & 0 & 0 & 0 \\
sexo opuesto & Relación & 0,4 & 0 & 25 & 0 \\
Ambos sexos & Atracción & 0 & 0 & 0 & 0 \\
por igual & Relación & 0 & 0 & 0 & 0 \\
Un poco más el & Atracción & 0 & 0 & 0 & 0 \\
mismo sexo & Relación & 0 & 0 & 0 & 0 \\
Principalmente & Atracción & 0 & 0 & 50 & 0 \\
mismo sexo & Relación & 0 & 0 & 25 & 0 \\
Solo mismo & Atracción & 0 & 50 & 0 & 0 \\
sexo & Relación & 0 & 50 & 0 & 0 \\
\hline
\end{tabular}


y coherentemente, solo ha tenido relaciones con personas del sexo opuesto. El otro 50\% dice sentirse atraído principalmente por personas de su mismo sexo si bien sólo la mitad de este porcentaje ha tenido realmente relaciones sexuales con personas de su mismo sexo. Por último, el $50 \%$ de quienes se identifican como asexuales ha tenido relaciones con personas del sexo opuesto y según indican se sienten atraídos por el sexo opuesto.

Si observamos estos datos en relación con el género podemos constatar que el 47,8\% de quienes se definen como heterosexuales son hombres y el 52,2\% mujeres. Esta proporción se repite tanto en personas que se definen como homosexuales (50\% son hombres y $50 \%$ mujeres), como en quienes se definen como asexuales (50\% son hombres y $50 \%$ mujeres) pero no en bisexuales que son en un $75 \%$ mujeres y en un $25 \%$ hombres. Sin embargo, las personas que se sienten atraídas solo por otras de su mismo sexo o principalmente por otras de su mismo sexo son todas mujeres. Esto significa que los hombres que se autodefinen como homosexuales no se caracterizan por un interés exclusivo por personas de su mismo sexo.

\section{Variables sociodemográficas}

Hemos querido observar también la distribución que este colectivo presenta en cuanto a una serie de variables. Por lo que se refiere a la religión, no hay relación entre las creencias religiosas y las distintas categorías. Como se puede observar en la tabla 2 un $50 \%$ de las personas que se definen como homosexuales son ateas pero el otro $50 \%$ son creyentes practicantes. Un $50 \%$ de las personas que se definen como bisexuales son ateas pero el otro $50 \%$ es creyente, aunque no practicante. Y las personas que se definen como asexuales son todas creyentes, el $50 \%$ practicantes y el otro $50 \%$ no. Solo quienes se definen como heterosexuales se reparten entre las tres categorías religiosas siendo en su mayoría creyentes no practicantes.

En cuanto a las restantes variables observadas, en la actualidad el 100\% de los homosexuales está activo sexualmente frente al $66,8 \%$ de los heterosexuales o el $25 \%$ de los bisexuales. Ninguna persona homosexual, bisexual o asexual usa Internet con fines sexuales, solo lo hacen quienes se definen como heterosexuales y en un porcentaje muy bajo $(16,7 \%)$, y

Tabla 2. Relación Orientación sexual autoinformada y religión (\%)

\begin{tabular}{lcccc}
\hline & Heterosexual & Homosexual & Bisexual & Asexual \\
\hline Ateo & 31,5 & 50 & 50 & 0 \\
Creyente no practicante & 51,7 & 0 & 50 & 50 \\
Creyente practicante & 16,8 & 50 & 0 & 50 \\
\hline
\end{tabular}


algo parecido ocurre con haber pagado por tener relaciones sexuales, lo cual solo lo hizo el 13,2\% de los heterosexuales. El uso de fantasías sexuales para incrementar la excitación es una práctica que realizan principalmente las personas bisexuales $(75 \%)$, seguidas por las heterosexuales $(53,9 \%)$ y las homosexuales $(50 \%)$ y que no realizan en ningún caso las personas asexuales. Por último, en cuanto a la fidelidad, el 50\% de los homosexuales y el 50\% de los bisexuales han sido infieles frente al $22,1 \%$ de los heterosexuales.

\section{Discusión}

La imagen que nos ofrecen los resultados acerca de la sexualidad de las personas mayores es mucho más compleja que la idea que nuestra sociedad tiene de ésta. Así, por ejemplo, el porcentaje de personas mayores que no se sienten atraídos exclusivamente por el sexo opuesto es casi del $13 \%$ ( $8,6 \%$ de hombres y $16,4 \%$ de mujeres) y un porcentaje cercano al $6 \%$ reconoce haber mantenido prácticas homoeróticas $(3,5 \%$ de hombres y $7,9 \%$ de mujeres).

La escasa investigación en este tema con personas mayores, hace que se carezca de estudios semejantes con dicha población, pero encontramos que nuestros resultados resultan coherentes con los obtenidos en otras edades. En un estudio que realizamos hace 25 años donde participaron 1135 sujetos con edades comprendidas entre los 14 y los 70 años, los resultados obtenidos mostraron que un 4,5\% (5,7\% hombres y $4 \%$ mujeres) de la muestra total había tenido alguna relación sexual con personas de su mismo sexo; el 7,7\% (4,3\% hombres, 9,9\% mujeres) lo había deseado en alguna ocasión; y el 18,4\% (12,5\% hombres y 22,9\% mujeres) había tenido fantasías de carácter homosexual alguna vez (Ballester y Gil, 1995). Y si comparamos con población joven de nuestro país, estudios recientes nos dan mayores porcentajes. Así, Ballester-Arnal y Gil-Llario (2016), hallaron que un 14,6\% de los jóvenes se consideraba homosexual o bisexual; y un porcentaje todavía mayor $(35,1 \%)$ se sentía atraído/a en mayor o menor medida por el mismo sexo.

Los resultados sobre diversidad afectivo-sexual que hemos obtenido en el presente estudio con personas mayores resultan muy interesantes por varios motivos. En primer lugar, porque dejan ver la existencia de una diversidad afectivo-sexual también en esta población. Lejos de la asunción, sin sentido por otra parte, de que la homosexualidad y bisexualidad solo afecta a los jóvenes, tenemos un porcentaje elevado de personas mayores que manifiestan sentirse atraídas en alguna medida por el mismo sexo. En segundo lugar, nos indica que las categorías referentes a la orientación sexual son insuficientes para captar la riqueza de la diversidad humana, dado que muchas de estas personas que se sienten atraídas en mayor o menor medida por el mismo sexo, se etiquetan, sin embargo, como heterosexuales. Esto también lo hemos hallado en población joven. En un estudio de Nebot-García, GarcíaBarba, Gil-Juliá, Giménez-García y Ballester-Arnal (2018) con participantes que tenían una media de 21 años, el 28,4\% de mujeres y el 18,5\% de hombres, a pesar de etiquetarse como heterosexuales, había sentido algún tipo de atracción sexual hacia personas de su mismo sexo.

En tercer lugar, en el presente estudio llama la atención cómo el porcentaje de mujeres que reconocen sentirse atraídas por el mismo sexo es el doble que el porcentaje de hombres, 
lo que puede estar relacionado con una mayor homofobia entre los hombres que entre las mujeres. Como aseguraba Elisabeth Badinter (1993), el hombre, para considerarse hombre, tiene que demostrar que no es mujer, que no es un niño y que no es homosexual y quizás eso explique por qué el hombre tiende a sentir más amenazada su frágil identidad masculina cuando reconoce sentirse atraído por otro hombre. Finalmente, estos datos nos tienen que alertar sobre la crueldad que implica invisibilizar socialmente la homosexualidad o bisexualidad en las personas mayores. Si ya de por sí, la sexualidad de este sector de la población suele estar invisibilizada y socialmente se castiga la manifestación pública de interés sexual por parte de hombres y mujeres mayores, en el caso de los que no son heterosexuales, esta invisibilización puede ser mucho mayor, dado que pertenecer a una minoría sexual a estas edades está más estigmatizado socialmente. Y eso puede llevar a que muchas de estas personas, al hacerse mayores, tengan que volver a "entrar en el armario" del que tanto les costó salir si es que alguna vez lo consiguieron hacer (Ballester-Arnal y Gil-Llario, 2019).

En el ámbito de la orientación sexual también es importante remarcar que hay un 2\% de las personas mayores que no se sienten atraídas ni por hombres ni por mujeres. Son lo que hoy denominaríamos "asexuales". Eso no significa que no puedan tener relaciones sexuales. Lo que significa es que, si las tienen, probablemente formen parte de una relación de pareja más basada en los sentimientos y emociones que en la atracción puramente sexual.

Los resultados obtenidos sobre la relación entre las creencias religiosas y el tipo de orientación sexual en la que se categorizan nuestros participantes, apuntan a una falta de influencia de estas creencias en la diversidad sexual de los mayores. Teniendo en cuenta la influencia de la religiosidad en el vínculo discriminación-significado de la vida (Moscardini et al., 2018), sería interesante seguir analizando cómo las creencias religiosas influenciaron su desarrollo sexual durante el transcurso de su vida hasta la edad adulta.

Si existe una conclusión a la que nos permiten llegar los resultados obtenidos en este estudio es que la heterosexualidad se mantiene como una categoría bastante compacta, aunque no tan extendida como se tiende a pensar, ya que las demás categorías parecen estar en evolución. Estos datos nos abren muchos interrogantes: ¿Hay personas homosexuales que todavía no han tenido relaciones con personas de su mismo sexo? ¿A qué ha podido deberse? ¿A cuestiones como las comentadas en la introducción, esto es, el temor al estigma (Boyer y Lorenz, 2020), a la presión recibida durante su desarrollo por parte de los padres (Fischer y Kalmijn (2020), a la oposición de los valores religiosos, etc.? ¿Han abandonado ya cualquier esperanza o su trayectoria vital les ha llevado a considerar más valiosos los aspectos que podrían perder que los que podrían ganar si intentaran hacer realidad sus deseos? ¿Por qué un porcentaje tan elevado de personas que solo han tenido relaciones sexuales con personas de su mismo sexo se definen como bisexuales? ¿Les resulta a las personas mayores menos problemático autodefinirse como bisexuales que como homosexuales a diferencia de lo que suele ocurrir con otros sectores de edad (Arriaga y Parent, 2019)? ¿Y cómo es que un porcentaje tan elevado de bisexuales sólo ha sentido atracción por personas del sexo opuesto? ¿Esto significa que su satisfacción sexual ha sido (y tal vez aún lo es) tan baja que consideran que quizá podrían encontrar lo que buscan en personas de su mismo sexo? ¿Y las personas que se definen como asexuales habiendo tenido relaciones y habién- 
dose sentido atraídas por personas del sexo opuesto, es, en esta misma línea argumental, porque su insatisfacción es tal que piensan que tal vez sean asexuales?

Todas estas cuestiones nos llevan a la necesidad de realizar estudios cualitativos que nos permitan recoger información más allá de la puramente cuantitativa que nos ofrecen los cuestionarios donde se pueda explorar el grado en el que la trayectoria vivida, esto es, la historia sexual, amorosa así como las inquietudes y el grado en que una persona, en términos de Erikson, siente que su vida ha tenido sentido, unido a las dificultades para experimentar placer en los términos en que se experimentaba en etapas evolutivas anteriores pueden estar explicando una realidad que, como se puede observar, no deja de evolucionar y crecer a lo largo de todo el ciclo vital.

Este estudio nos aporta información relevante sobre la diversidad sexual en las personas mayores, tema poco investigado, pero de gran relevancia para este colectivo (FredtiksenGoldsen \& Kim, 2015). Esto nos ayudará a entender mejor su situación, a visibilizar su vivencia y detectar aspectos que sería interesante incluir en los programas de sexualidad dirigidos a las personas mayores.

No obstante, hay que remarcar como limitaciones del estudio, que sería adecuado incrementar el número de participantes para obtener así unos resultados más concluyentes, y que permitiera el análisis por distintos intervalos de edad, para observar si existen diferencias.

\section{Referencias}

Alonso-Sánchez, J. y Muyor-Rodríguez, J. (2020). La homosexualidad en las personas mayores: vivenciando narrativas en torno a las discriminaciones y prácticas resilientes. Trabajo Social Global, 10(18), 46-71. 10.30827/ tsg-gsw.v10i18.8654

Arriaga, A.S. y Parent, M.C. (2019). Partners and Prejudice: Bisexual Partner Gender and Experiences of Binegativity From Heterosexual, Lesbian, and Gay People. Psychology of Sexual Orientation and Gender Diversity, 6(3), 382-391 http://dx.doi.org/10.1037/sgd0000337

Badinter, E. (1993). XY, la Identidad masculina. Madrid: Alianza Editorial.

Ballester, R. y Gil, M. D. (1995). Homosexualidad: comportamientos deseos y fantasías. Análisis y modificación de Conducta, 21, 264-284.

Ballester-Arnal, R. y Gil-Llario, M.D. (2016). Orientación del deseo sexual: Crisis y decadencia de los modelos categoriales. En A. López de la LLave (Coord.), Sexología positiva: Placer, salud y bienestar (pp. 59-66). Madrid: Universidad Nacional de Educación a Distancia.

Ballester-Arnal, R. y Gil-Llario, M.D. (2019). Orientación sexual, prácticas, frecuencia, fantasías y uso de la pornografía. En R. Ballester-Arnal, M.D. Gil-Llario, C. Giménez, E. Ruiz, B. Gil, J. Castro y V. Morell. Sexualidad y calidad de vida en las personas mayores: análisis de la población de Castellón (pp. 39-58). Castellón: Fundación Dávalos-Fletcher.

Boyer, S.J. y Lorenz, T.K. (2020). The Impact of Heteronormative Ideals Imposition on Sexual Orientation Questioning Distress. Psychology of Sexual Orientation and Gender Diversity. 7(1), 91-100. http://dx.doi. org/10.1037/sgd0000352

Ceballos-Fernández, M. (2014). Identidad homosexual y contexto familiar heteroparental: implicaciones educativas para la subversión social. Revista Latinoamericana de Ciencias Sociales, Niñez y Juventud, 12(2), 643-658. http://dx.doi.org/10.11600/1692715x.1229140514 
Fredriksen- Goldsen, K.I. \& Kim, H.J. (2015). Count me in: Response to sexual orientation measures among older adults. Research on Aging, 37(5), 464-480.

Fischer, M. M. y Kalmijn, M. (2020, May 14). Do Adult Men and Women in Same-Sex Relationships Have Weaker Ties to Their Parents? Journal of Family Psychology. Advance online publication. http://dx.doi.org/10.1037/ fam0000696

Herz, M. y Johansson, T. (2015). The normativity of the concept of heteronormativity. Journal of Homosexuality, 62(8), 1009-1020. https://doi.org/10.1080/00918369.2015.1021631

Hogg, M. A. (2007). Uncertainty-identity theory. In M. P. Zanna (Ed.), Advances in experimental social psychology (pp. 69-126). San Diego, CA: Elsevier Academic Press.

Hogg, M. A. (2012). Uncertainty-identity theory. En P. A. M. van Lange, A. W. Kruglanski, y E. T. Higgins (Eds.), Handbook of theories of social psychology (pp. 62-80). Los Ángeles, CA: SACE. http://dx.doi. org/10.4135/9781446249222.n29

Kinsey, A., Pomeroy, W. y Martin, C. (1948). Sexual behavior in the human male. Philadelphia: Saunders.

Klein, F. (1978). The Bisexual Option: A Concept of One Hundred Percent Intimacy. EE UU: Arbor house.

Moscardini, E.H., Douglass, R.P., Conlin, S.E. y Duffy, R.D. (2018). Minority Stress and Life Meaning Among Bisexual Adults: The Role of Religiosity. Psychology of Sexual Orientation and Gender Diversity, 5(2), 194-203. http://dx.doi.org/10.1037/sgd0000284

Nebot-García, J.E., García-Barba, M., Gil-Juliá, B., Giménez-García, C. y Ballester-Arnal, R. (2018). Comportamientos homosexuales en jóvenes heterosexuales: diferencias de género. Àgora de Salut, 5, 59-68

Pachankis, J.E. y Bränström, R. (2018). Hidden From Happiness: Structural Stigma, Sexual Orientation Concealment, and Life Satisfaction Across 28 Countries. Journal of Consulting and Clinical Psychology, 86(5), 403-415. http://dx.doi.org/10.1037/ccp0000299 